\title{
EFFICACY OF ORAL ANTI-DIABETIC AGENTS FOR GLYCAEMIC CONTROL IN TYPE-2 DIABETIC PATIENTS WITH ACID PEPTIC DISEASE
}

\author{
Vineeth Reddy G ${ }^{1}$, Anila Reddy T1, Venkateshwarlu Eggadi' ${ }^{1}$, Sharavanabhava B.S ${ }^{*}$ \\ ${ }^{1}$ Department of Clinical Pharmacy \& Pharm.D., Vaagdevi College of Pharmacy, Warangal, Telangana, Kakatiya \\ University- 506001, India. \\ *Corresponding author e-mail: sharvanabhava6@gmail.com
}

\begin{abstract}
:
Diabetes is a chronic disease requiring intensification of therapy as it progresses. The goal of many clinicians who manage diabetes is to achieve glycaemic control and a minimum number of hypoglycaemic episodes. The present study is designed to evaluate the better glycaemic control efficiency of Metformin and its combination with Glimepiride and Pioglitazone in patients with acid peptic disease along with diabetes. A total of 82 patients were assigned in to two groups i.e., group of patients with Diabetes only group and group of patients with Diabetes and Acid Peptic disease. In this work various parameters Weight, BMI, SBP, DBP, $\mathrm{Hb}$, Serum creatinine, FBS, PLBS, $\mathrm{HbA}_{1} \mathrm{c}$, Serum Cholesterol, HDL, LDL, VLDL, CHOL/HDL ratio, LDL/HDL ratio, TG were measured to investigate and to compare the efficacy of three treatment regimens to achieve durable glycaemic control in patients suffering with diabetes along with complications (Acid peptic disease) who were undergoing treatment i.e. monotherapy with Metformin, combination therapy (Metformin and Pioglitazone, Metformin and Glimepiride). Results of the present study showed that in Diabetic only group the three regimens decreases FBS and PLBS levels where as in diabetic and acid peptic disease patients the treatment with Metformin and Pioglitazone showed significant glycaemic control.
\end{abstract}

Key words: Diabetes, Acid Peptic disease, Metformin, Pioglitazone, Glimepiride.

\section{INTRODUCTION:}

Diabetes mellitus is a disease characterized by hyperglycemia, depletion of antioxidants, and alteration in lipid metabolism. Diabetes is characterized by increased generation of reactive oxygen species and decreased antioxidant levels in the body. It is associated with an increased prevalence of gastrointestinal tract symptoms. Diabetics have increased vulnerability of the gastric mucosa to various ulcerogens such as ethanol, ischemia/reperfusion, stress, and non steroidal anti-inflammatory drugs. The notable changes that are often observed in patients with chronic diabetes mellitus are decreased gastric inflammation and ulcer disease occur with high prevalence in patients with type 2 diabetes mellitus. However, peptic ulcers related to diabetes mellitus are often associated with complications such as gastrointestinal bleeding [1]. Certain GI manifestations of DM are linked to diabetic autonomic neuropathy. This condition increases with age, duration of DM, and the presence of peripheral neuropathy. Autonomic neuropathy affects the sympathetic and parasympathetic innervations of the bowel [2].

Diabetic patients are more prone and at risk to acquire Helicobacter pylori (H.pylori) infection. Therefore proper monitoring of blood glucose level and screening for H. pylori infection are effective preventive measures 
for this life threatening infection. The role of Helicobacter pylori infection in diabetic dyspepsia is mainly related to blood glucose concentration. Hyperglycemia may induce the infection by $\mathrm{H}$. pylori or the silent infection may get reactivated and produce symptoms of dyspepsia in diabetes [3]. The pathogenesis, incidence, complication rates, response to acid suppression and $H$. pylori eradication therapy in peptic ulcer associated with chronic disease such as liver cirrhosis, chronic renal failure, diabetes mellitus, and critically ill conditions are different from those with general population, so that the management strategies also should be differentiated [4]. The study suggests that diabetic patients are at more risk for $H$. pylori infection in comparison to non-diabetic population so every diabetic patient with acid peptic disorder must be screened for H. pylori. There is a dire need to provide proper counseling, education and awareness regarding diabetic mellitus and its association with H. Pylori infection. Effective and appropriate measures should be taken against control of diabetes mellitus, eradication of H. pylori infection [4].

\section{MATERIALS AND METHODS}

The present study was conducted in Diabetic patients visiting Sri Bhadrakali Diabetic Hospital, Hanamkonda, and Warangal. The work Protocol was submitted and got approved by the Human Ethics Committee [IHEC/JCP/12/001]. Informed written consent was obtained from all the patients.

A total of 82 patients, aged 30-65 years were assigned in to 2 groups according to their complications based on medical history. All the patients were diabetic (type 2 diabetes). Among these 32 patients are with Acid Peptic disease. In this patient population some patients received monotherapy with Metformin and some of them received www.ijapbjournal.com combination therapy i.e., Metformin and Glimepiride and Metformin and Pioglitazone.

Study Criteria:

Inclusion Criteria: Patients of either sex, age group between 30-65 years and diagnosed with type 2 diabetes. Patients diagnosed with Acid Peptic disease as other complication along with type 2 diabetes. Patients who were on treatment with Metformin monotherapy and combination therapy with Glimepiride or Pioglitazone.

Exclusion Criteria: Patients receiving only insulin. Pregnant and lactating patients, Pediatrics, Patients who were very weak and with severe disability, Patients with other comorbid diseases like tuberculosis, asthma or any other diseases under polymorphism. Patients were excluded if they had a history of type 1 diabetes and secondary forms of diabetes.

\section{RESULTS}

In this study, diabetic patients with Acid Peptic disease aged between 30 to 65 years were enrolled. All the patients were followed up for 9 months. Descriptive statistics of the study groups are explained below.

\section{Effect of Drugs on Various Parameters:}

There was no significant change in body weight and BMI in all three visits in patients with Metformin alone and in combination with Glimepiride and Pioglitazone groups.

Systolic B.P. was not changed in all three visits in patients taking Metformin and Glimepiride, where as in patients taking Metformin + Pioglitazone, it was slightly reduced from $124.25 \pm 8.26$ to $123.83 \pm 5.70 \mathrm{~mm} \mathrm{Hg}$ and in case of patients taking Metformin alone systolic blood pressure was reduced on second visit but later increased on third visit.

Diastolic B.P. was slightly increased in all three visits in patients treated with IJAPB 
Metformin in combination with Glimepiride and Pioglitazone. In patients taking Metformin alone it was reduced from $81.20 \pm 9.18$ to $77.5 \pm 8.86 \mathrm{~mm} \mathrm{Hg}$ but later increased to $81.40 \pm 5.42 \mathrm{~mm} \mathrm{Hg}$ on their third visit.

Haemoglobin levels were not changed in all three visits in patients treated with Metformin alone. But in patients treated with Metformin and Glimepiride combination the levels were slightly elevated from $11.46 \pm 1.32$ to $12.17 \pm 1.39 \mathrm{gm} \%$. Hb levels were slightly increased $(\mathrm{p}<0.01)$ in second visit in patients treated with Metformin and Pioglitazone when compared with Metformin alone.

Serum creatinine levels were significantly decreased from $1.15 \pm 0.29$ to $0.87 \pm 0.13$ $\mathrm{mg} / \mathrm{dl}, 1.29 \pm 0.31$ to $0.98 \pm 0.22 \mathrm{mg} / \mathrm{dl}, 1.50$ \pm 0.31 to $0.90 \pm 0.20 \mathrm{mg} / \mathrm{dl}$ in their third visit in patients taking Metformin alone and in combination with Glimepiride and with Pioglitazone respectively.

FBS was decreased from $125 \pm 19.87$ to $104.3 \pm 11.44,153.5 \pm 36.55$ to $117.7 \pm$ $22.38 \mathrm{mg} / \mathrm{dl}, 144.58 \pm 32.76$ to $113.42 \pm$ $17.29 \mathrm{mg} / \mathrm{dl}$ in their third visit in patients treated with Metformin alone, with Metformin + Glimepiride and with Metformin + Pioglitazone combinations respectively.

Post prandial blood sugar levels were decreased from $181.50 \pm 30.97$ to $147.90 \pm$ $21.29 \mathrm{mg} / \mathrm{dl}, 223.60 \pm 47.28$ to $153.4 \pm$ $17.38 \mathrm{mg} / \mathrm{dl}$ in patients treated with Metformin alone and in combination with Glimepiride on their third visit. PBS levels were significantly elevated $(p<0.05)$ in the first visit of patients treated with Metformin and Pioglitazone combination, but these levels were reduced gradually from first visit to third visit ( $239 \pm 57.8$ to $161.17 \pm 28.4$ $\mathrm{mg} / \mathrm{dl}$ ) .

HbA1c slightly reduced from $8.51 \pm 1.22$ to $7.35 \pm 0.89$ and $9.24 \pm 1.38$ to $7.87 \pm 0.73$ on their third visit in patients treated with
Metformin alone and in combination with Pioglitazone respectively. But in patients treated with Metformin and Glimepiride HBA1c levels were raised from 7.34 \pm 0.9 to $8.46 \pm 0.78$ on their second visit and then reduced to $7.87 \pm 0.70$ on their third visit.

Serum cholesterol levels were decreased from $241.7 \pm 58.05$ to $201.3 \pm 43.32 \mathrm{mg} / \mathrm{dl}$, $220.8 \pm 62.9$ to $179 \pm 35.25 \mathrm{mg} / \mathrm{dl}$ and $201 \pm$ 40.36 to $170.83 \pm 31.72 \mathrm{mg} / \mathrm{dl}$ on their third visit in patients taking Metformin alone in combination with Glimepiride and Pioglitazone respectively .

HDL levels were raised from $43.7 \pm 5.96$ to $46.9 \pm 7.39 \mathrm{mg} / \mathrm{dl}$ and $44.7 \pm 6.39$ to $45.7 \pm$ $9.49 \mathrm{mg} / \mathrm{dl}$ on their third visit in patients treated with Metformin and in combination with Glimepiride. But in patients treated with Metformin in combination with Pioglitazone, HDL levels reduced from $42.92 \pm 7.72$ to $37.75 \pm 3.11 \mathrm{mg} / \mathrm{dl}$ on their second visit, but later raised to $42.42 \pm 6.85 \mathrm{mg} / \mathrm{dl}$ on their third visit.

LDL levels were decreased from 164.1 \pm 50.79 to $128.58 \pm 41.12 \mathrm{mg} / \mathrm{dl}, 140.92 \pm 58.77$ to $105.14 \pm 30.16 \mathrm{mg} / \mathrm{dl}$ and $121.77+/-37.41$ to $98.95+/-30.97 \mathrm{mg} / \mathrm{dl}$ in their third visit in patients treated with Metformin alone and in combination with Glimepiride, Pioglitazone respectively.

VLDL levels were decreased from 33.66+/5.54 to $27.36 \pm 5.78 \mathrm{mg} / \mathrm{dl}, 36.32 \pm 12.06$ to $29.38 \pm 6.26 \mathrm{mg} / \mathrm{dl}$ on their third visit in patients treated with Metformin alone, combination with Pioglitazone .In patients treated with Metformin in combination with Glimepiride VLDL levels were slightly increased from $35.18 \pm 10.99$ to $36.46 \pm$ $7.58 \mathrm{mg} / \mathrm{dl}$ on their second visit but later levels were reduced to $28.16 \pm 5.31 \mathrm{mg} / \mathrm{dl}$.

The ratio of $\mathrm{CHO}$ to $\mathrm{HDL}$ was reduced from $6.24 \pm 1.32$ to $4.47 \pm 1.15,5.74 \pm 1.64$ to $4.45 \pm 1.28,5.35 \pm 1.13$ to $4.23 \pm 0.74$ on their third visit in all patients taking Metformin 
Table 1: Parameters (Mean \pm SD) during Visit1, 2, and 3 after a treatment with Metformin, Metformin \& Glimepiride and Metformin \& Pioglitazone in Diabetic and Acid peptic patients.

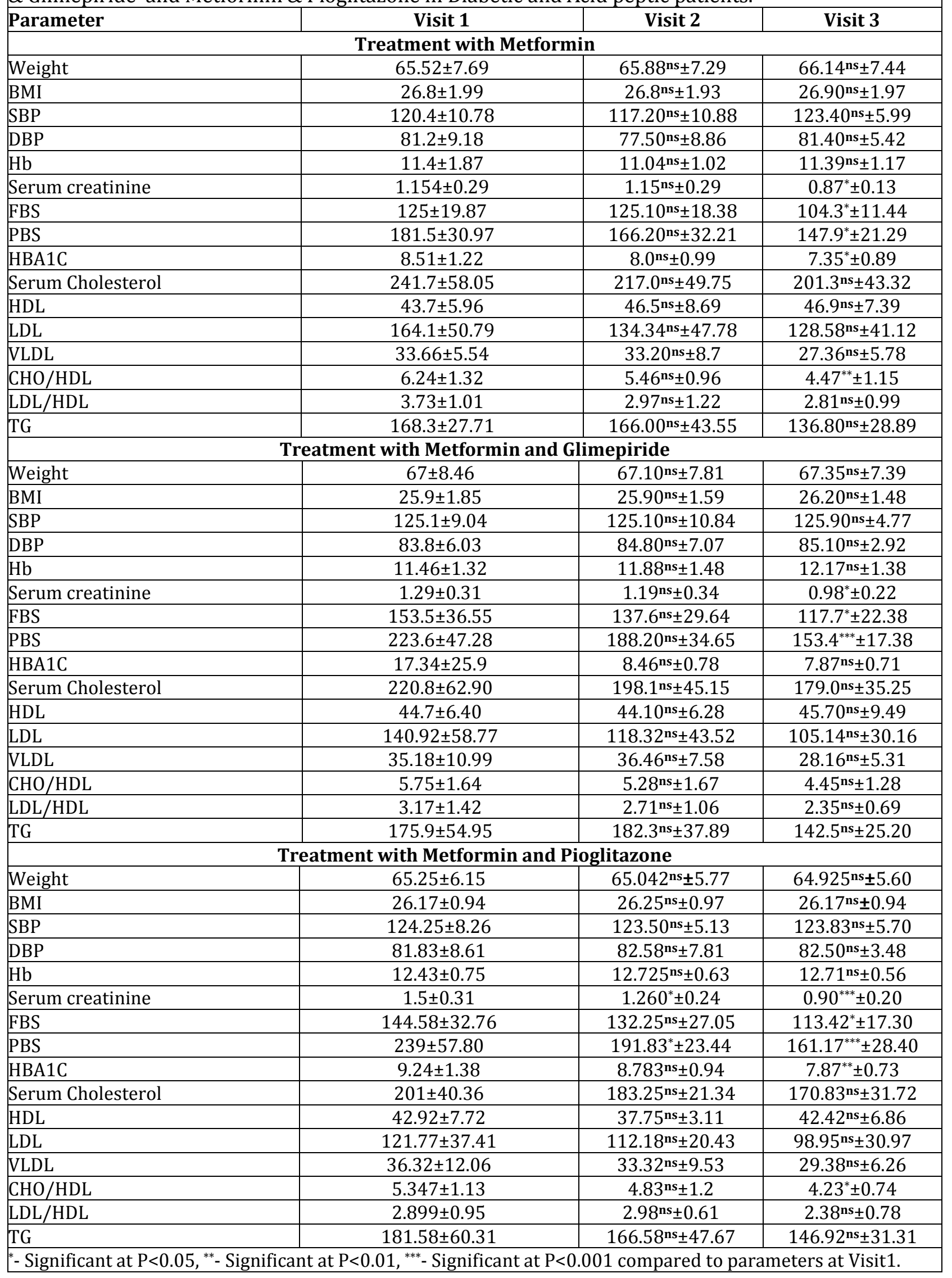


alone, Metformin with Glimepiride and with Pioglitazone respectively .

LDL/HDL ratio was reduced from $3.73 \pm 1.01$ to $2.81 \pm 0.99$ and $3.17 \pm 1.42$ to 2.35 \pm 0.69 on their third visit in patients taking Metformin alone and Metformin with Glimepiride respectively. Patients taking Metformin+Pioglitazone combination the ratio was raised from $2.89 \pm 0.95$ to $2.98 \pm$ 0.61 on their second visit but later reduced to $2.38 \pm 0.78$.

TG levels were decreased from $168.3 \pm 27.71$ to $136.8 \pm 28.86 \mathrm{mg} / \mathrm{dl}$ and $181.58 \pm 60.31$ to $146.92 \pm 31.31 \mathrm{mg} / \mathrm{dl}$ and on their third visit in patients treated with Metformin alone and in combination with Pioglitazone. In patients treated with Metformin and Glimepiride combination TG levels were raised from $175.90 \pm 54.95$ to $182.3 \pm 37.89 \mathrm{mg} / \mathrm{dl}$ on their second visit , but later levels reduced to 142.5 $\pm 25.20 \mathrm{mg} / \mathrm{dl}$.

By treatment with Metformin significant difference $(p<0.05)$ in serum creatinine, fasting blood sugar, post lunch blood sugar during visit3, significant difference $(\mathrm{p}<0.01)$ LDL/HDL ratio during visit 3 when compared to visit1.

By treatment with Metformin and Glimepiride there was a significant difference $(\mathrm{p}<0.05)$ in serum creatinine, fasting blood sugar during visit3, and significant difference $(\mathrm{p}<0.001)$ in post lunch blood sugar during visit3 when compared to visit1.

By treatment with Metformin and Pioglitazone there was a significant difference $(\mathrm{p}<0.05)$ serum creatinine, post lunch blood sugar during visit2, fasting blood sugar and cholesterol/HDL ratio during visit3, significant difference $(\mathrm{p}<0.01)$ in $\mathrm{HbA1C}$ during visit3 and LDL during visit 2 and significant difference $(\mathrm{p}<0.001)$ in serum creatinine, post lunch blood sugar during visit3 when compared to visit 1.

\section{DISCUSSION} doi.org/10.38111/ijapb.20160203001

Few studies evaluated for potential influence of Helicobacter pylori for the evaluated levels of HbA1c and have been seen correlation between elevated levels of HbA1c and H.Pylori. Similarly in the current study Metformin monotherapy, Metformin with Glimepiride and Metformin with Pioglitazone were unable to reduce elevated HbA1c level in patients with acid peptic disease, maintaining all other parameters in their optimal range [5].

They were also unable to reduce LDL levels irrespective of they were on Rabeprazole therapy.

By the treatment of diabetic-acid peptic disease patients with Metformin and in combination of Metformin with Glimepiride and Pioglitazone in Metformin and Glimepiride treated groups there was $22.8 \%$ increase in FBS, 23.19\% increase in PBS, $103.76 \%$ increase in HbA1c, $14.12 \%$ reduction in serum cholesterol, $15.03 \%$ reduction in LDL/HDL ratio when compared to Metformin alone treated groups. Whereas in Metformin and Pioglitazone treated groups there was $15.66 \%$ increase in FBS, $16.58 \%$ reduction in serum cholesterol, $16.49 \%$ reduction in LDL levels, $22.29 \%$ reduction in LDL/HDL ratio when compared to Metformin alone treated groups.

\section{CONCLUSION}

In this study various parameters like Weight, BMI, SBP, DBP, Hb, Serum creatinine, FBS, PLBS, HbA1c, Serum Cholesterol, HDL, LDL, VLDL, CHOL/HDL ratio, LDL/HDL ratio, TG were measured to investigate and to compare the efficacy of three treatment regimens i.e., Metformin monotherapy , Metformin with Glimepiride and Metformin with Pioglitazone in Diabetic patients, Diabetic patients with Acid Peptic disease. 
Vineeth Reddy $G$ et al.

In conclusion, treatment with Metformin and Pioglitazone combination in diabetic and acid peptic disease patients showed significant glycaemic control than the other two groups.

\section{ACKNOWLEDGEMENT}

Authors are thankful to the Secretary, Dr.Ch.Devender Reddy, Viswambhara Educational Society, Warangal, and Telangana for giving us opportunity to work and providing necessary facilities to carry out this Research work.

\section{CONFLICT OF INTEREST}

The author(s) confirm that this article content has no conflict of interest.

\section{REFERENCES}

[1] D.U. Owu, A.O. Obembe, C.R. Nwokocha, I. E. Edoho, E. E. Osim. Gastric Ulceration in Diabetes Mellitus: Protective Role of Vitamin C. International Scholarly Research Network ISRN Gastroenterology Volume 2012, Article ID 362805, 7 pages doi:10.5402/2012/36280
Int. J. Adv. Pharm. Biotech., 2016; 2(3): 1-6 doi.org/10.38111/ijapb.20160203001

[2] Elam M, Thoren P, Svensson TH. Locus coeruleus neurons and sympathetic nerves: Activation by visceral afferents. Brain Res. 1986; 375:117-125.

[3] Bikha Ram Devrajani, Syed Zulfiquar Ali Shah, Aftab Ahmed Soomro, Tarachand Devrajani. Type 2 diabetes mellitus: A risk factor for Helicobacter pylori infection: A hospital based case-control study. Int J Diabetes Dev Ctries. 2010 Jan-Mar; 30(1): 22-26.

[4] Ji Hyun Kim 1, Jeong Seop Moon, Sam Ryong Jee, Woon Geon Shin, Soo-Heon Park. Guidelines of treatment for peptic ulcer disease in special conditions. Korean J Gastroenterol. 2009;54(5):318-27.

[5] Yu Chen, and Martin J. Blaser. Association Between Gastric Helicobacter pylori Colonization and Glycated Hemoglobin Levels. The Journal of Infectious Diseases •2012; 205:1195-1201.

\section{How to cite this article:}

Vineeth Reddy $G$ et al Efficacy of Oral Anti-Diabetic Agents For Glycaemic Control In Type-2 Diabetic Patients With Acid Peptic Disease. Int. J. Adv. Pharm. Biotech., 2016; 2(3): 1-6. 